\title{
Treating a Sexual Abuse Trauma with Eye Movement Desensitisation and Reprocessing Therapy in an Adult Community Mental Health Team Setting, A Case Series
}

\author{
${ }^{* 1}$ Jonathan Hutchins and ${ }^{2}$ Clare Mason \\ ${ }^{1}$ Clinical Director Hutchins Psychology Services, UK \\ ${ }^{2}$ Honorary Research Assistant, Hutchins Psychology Services, UK
}

Submission:August 08, 2017; Published: August 24, 2017

*Corresponding author: Jonathan Hutchins, Clinical Director Hutchins Psychology Services Ltd, 16 Chequers Street, St Albans, AL1 3YD, UK, Tel: 7725363481; Email: jonathan@hutchinspsychology.co.uk

\section{Abstract}

Introduction: Rape and sexual trauma are two of the most prevalent traumatic experiences linked to mental health problems. A challenge exists in the treatment of such traumas. This study focuses on three case studies treating sexual trauma using Eye Movement Desensitisation and Reprocessing Therapy (EMDR).

Method: This was a case series design using pre- and post-measures including the BDI, BAI, OQ.45 and IES-R with three different clients. A three month follow - up was also completed.

Results: EMDR therapy was effective at treating sexual abuse traumatic experiences within a short number of sessions. Recovery was shown on all four outcome measures.

Discussion: Although this is a case series study, it provides some evidence of EMDR being an effective treatment for sexual abuse trauma. The additional advantages of EMDR are discussed, including the strength of not having to talk about the trauma in-depth within the therapy.

Abbreviations: tfCBT: Trauma Focused Cognitive Behavioural Therapy; EMDR: Eye Movement Desensitization and Reprocessing Therapy; BDI: Beck Depression Inventory; BAI: Beck Anxiety Inventory OQ.45: Outcome Questionnaire-45; IES-R: Impact of Events Scale-Revised.

\section{Introduction}

Rape and sexual trauma are two of the most prevalent traumatic experiences both in childhood [1] and as adults [2]. Often victims experience high levels of shame and self-blame which can prevent them from accessing psychological therapy [3]. The experience of shame, combined with the natural fear of talking about a trauma, can also be significant impediments to psychological treatment. Currently the NICE guidelines [4] and recent meta analytical studies [5] recommend both trauma focused Cognitive Behavioural Therapy (tfCBT) and Eye Movement Desensitization and Reprocessing Therapy (EMDR) for treatment of Post-Traumatic Stress Disorder. Both tfCBT and EMDR utilise the theory that PTSD results from the inadequate processing of a trauma, due to the individual dissociating at the time. This is thought to be because their experience of the event is over whelming [6,7]. Traumafocused CBT requires the individual to describe the trauma in detail, reliving the experience with the therapist and aiming to find hotspots within the trauma memory which can then be addressed with cognitive therapy techniques [8]. This approach can be very challenging, especially for victims of rape and sexual abuse trauma, due to the significant levels of shame they may feel [9]. One particular advantage of EMDR is that it does not require the victim to describe the trauma in depth. In fact, some EMDR protocols actively encourage the traumatic experience be blind to the therapist [10]. This may be very helpful for the 
victim as it can significantly reduce the shame and self-blame associated with their experience [10]. As a result, victims are more willing to engage, leading to more positive outcomes. This article highlights three case studies using EMDR, within a UK National Health Service (NHS) community mental health team.

\section{Method \\ Design}

This is a case series design, based on EMDR intervention with three clients. The outcome measures were the service standard within the locality in which the clients were seen. They consisted of the Beck Depression Inventory (BDI) [11]; Beck Anxiety Inventory (BAI) [12] the Outcome Questionnaire 45item version (OQ.45) (a measure of an individual's functioning) [13], a measure of an individual's functioning and the Impact of Events Scale Revised (IES-R) (a measure of Post-Traumatic Stress Disorder) [14]. These measures were assessed pre- and post- treatment and at 3 months follow-up. Where there were multiple traumas clients were asked to describe and initially focus on the most dominant trauma when completing the IES-R. Clients were then asked to complete an IES-R for each individual trauma. Therefore, in the case of Client 2, six IES-R's were gathered; pre- and post-treatment as six sexual traumatic events were being treated.

\section{Consent}

All clients gave their verbal and written consent for the case study.

\section{Client Details:}

Client 1 was a 60- year-old man, referred by his GP to the local community mental health team for depression and cognitive behavioral therapy. On assessment with the Clinical Psychologist he disclosed that he had been sexually abused by his uncle from the age of 7 to 11 . He had experienced nightmares and flashbacks about the events all his life. He also reported a significant alcohol problem, consuming up to four pints of beer daily. Although there were considerable problems with the client's drinking, the treating clinician decided to fast-track the client into trauma focused work, on the agreement that the client would not drink before the session. The clinician believed that if the trauma could be treated, the client's avoidant behavior with alcohol may cease.

Client 2 was a 22-year-old woman already under the care of the community mental health team. She had previously been diagnosed with Borderline Personality Disorder, with difficulties regulating her emotions and interpersonal relationships. She was referred by the team psychiatrist due to high anxiety. On assessment with the Clinical Psychologist, she reported a complex sexual trauma history which included being sexually abused by her next door neighbor from the age of 10 to 15 . In addition, she reported several experiences of being raped at her workplace between the ages of 16 and 19. She reported nightmares and flashbacks about these events which had occurred in several different places. The Clinical Psychologist accepted her for trauma focused therapy as, despite her previous diagnosis of BPD, she met the suitability criteria in terms of being able to self-sooth and having no risk behaviors present at the time of the intervention.

Client 3 was a 35-year-old woman, referred by her GP to the community mental health team for treatment of anxiety and depression. She was assessed by the team Clinical Psychologist, at which point she disclosed that she had been raped by a coworker at a restaurant toilet when she was 19. She had regular intrusions about the event ever since.

\section{Treatment}

The participants were seen by a Clinical Psychologist who is an Accredited EMDR Europe Practitioner, with over three years' experience of using EMDR. The treating clinician was supervised by an EMDR Europe Accredited Consultant. EMDR was chosen for several reasons including the clients desire to avoid talking about the traumatic events in depth, which would have been required in a tfCBT approach. In addition, EMDR was also thought to be preferable given the multiple traumatic events to be addressed in a short time frame. EMDR has been shown to be quick and effective in the treatment of PTSD and complex trauma cases [15]. Treatment followed the standard EMDR protocol [16].

In the case of Client 2, multiple traumatic experiences were identified. Some of these were the same sexual traumatic event that had occurred repeatedly over several years. Six discrete trauma memories and several composite memories were identified Following standard EMDR protocol, these cases were treated in a single event approach. It is thought that where the same event is repeated, in re-processing one trauma memory of the experience, they are all treated [15]. Six discrete trauma memories and several composite memories were identified. Where a traumatic event was addressed within a single session, IES-R was recorded on a weekly basis, for a period of six sessions. Therefore, six pre-and post-IES-R's were recorded for this case.

\section{Results}

\section{Client 1: Three Sessions}

The outcome measures for the three-session intervention are shown below. The intervention consisted of one assessment session, including stabilization of the client, one EMDR processing session and one follow-up session. The client reported significant improvements at follow-up, both in terms of the memory no longer being disturbing to him and also in his relationships, where he felt closer to his children and wife (Table 1). 


\section{Psychology and Behavioral Science International Journal}

Table 1: Client 1, Three Sessions. All measures demonstrate a reduction in symptoms at post-treatment and 3 months follow-up

\begin{tabular}{|c|c|c|c|c|c|c|}
\hline \multicolumn{2}{|c|}{ Outcome Measure } & Pre-treatment & Post-treatment & 3 Month Follow Up & Cut off & Reliable Change \\
\hline OQ.45 Subscales & Symptom Distress & 55 & 20 & 10 & 36 & Recovered \\
\hline & $\begin{array}{l}\text { Interpersonal } \\
\text { Relations }\end{array}$ & 20 & 8 & 0 & 15 & Recovered \\
\hline & Social Role & 19 & 6 & 0 & 12 & Recovered \\
\hline \multicolumn{2}{|c|}{ OQ45.2 Total } & 94 & 34 & 10 & 63 & Recovered \\
\hline \multicolumn{2}{|c|}{ BDI-II } & 36 & 14 & 6 & 36 & Recovered \\
\hline \multicolumn{2}{|c|}{ BAI } & 30 & 10 & 2 & None & Recovered \\
\hline \multicolumn{2}{|c|}{ IES-R } & 55 & 8 & 0 & 33 & Recovered \\
\hline
\end{tabular}

\section{Client 2: Eight Sessions, Six Traumas}

The outcome measures highlighted below, show significant gains in symptom reduction on both the Beck Anxiety and Depression scales. In addition, on measuring each individual

Table 2: Client 2, Six Traumas, Eight Sessions.

\begin{tabular}{|c|c|c|c|c|c|c|}
\hline \multicolumn{2}{|c|}{ Outcome Measure } & Pre-treatment & Post-treatment & 3 Month Follow Up & Cut off & Reliable Change \\
\hline OQ.45 Subscales & Symptom Distress & 80 & 45 & 30 & 36 & Recovered \\
\hline & $\begin{array}{c}\text { Interpersonal } \\
\text { Relations }\end{array}$ & 24 & 12 & 5 & 15 & Recovered \\
\hline & Social Role & 18 & 8 & 4 & 12 & Recovered \\
\hline \multicolumn{2}{|c|}{ OQ45.2 Total } & 122 & 65 & 39 & 63 & Recovered \\
\hline \multicolumn{2}{|c|}{ BDI-II } & 30 & 16 & 10 & 36 & Recovered \\
\hline \multicolumn{2}{|c|}{ BAI } & 40 & 12 & 9 & None & Recovered \\
\hline \multicolumn{2}{|c|}{ IES-R Trauma 1} & 70 & 20 & 4 & 33 & Recovered \\
\hline \multicolumn{2}{|c|}{ IES-R Trauma 2} & 75 & 18 & 7 & 33 & Recovered \\
\hline \multicolumn{2}{|c|}{ IES- R Trauma 3} & 65 & 10 & 0 & 33 & Recovered \\
\hline \multicolumn{2}{|c|}{ IES-R Trauma 4} & 55 & 15 & 2 & 33 & Recovered \\
\hline \multicolumn{2}{|c|}{ IES-R Trauma 5} & 40 & 8 & 0 & 33 & Recovered \\
\hline \multicolumn{2}{|c|}{ IES-R Trauma 6} & 50 & 7 & 0 & 33 & Recovered \\
\hline
\end{tabular}

Table 3: Client 3, Five Sessions. All measures demonstrate a reduction in symptoms at post-treatment and 3 months follow-up

\begin{tabular}{|c|c|c|c|c|c|c|}
\hline \multicolumn{2}{|c|}{ Outcome Measure } & Pre-treatment & Post-treatment & 3 Month Follow Up & Cut off & Reliable Change \\
\hline OQ.45 Subscales & Symptom Distress & 60 & 30 & 15 & 36 & Recovered \\
\hline & $\begin{array}{c}\text { Interpersonal } \\
\text { Relations }\end{array}$ & 30 & 10 & 8 & 15 & Recovered \\
\hline Social Role & 15 & 8 & 2 & 63 & Recovered \\
\hline OQ45.2 Total & 105 & 48 & 0 & 36 & Recovered \\
\hline BDI-II & 25 & 10 & 0 & None & Recovered \\
\hline BAI & 30 & 6 & 0 & 33 & Recovered \\
\hline IES-R & 38 & 5 & & 25 & & \\
\hline
\end{tabular}

\section{Client 3: Five Sessions}

The outcome measures Table 3 highlight symptom improvement following five sessions of EMDR addressing the client's single-event rape trauma. Substantial improvements traumatic experience using IES- $\mathrm{R}$, there were significant reductions in PTSD symptoms one week after EMDR reprocessing treatment. At this point, each memory no longer met criteria for PTSD. These gains were maintained at follow-up (Table 2). 


\section{Discussion}

The results of these three case studies suggest that EMDR is a highly effective and efficient treatment for sexual trauma, both in terms of childhood sexual abuse and adult rape. Reliable change is highlighted across all outcome measures, to the point where all three clients no longer met the diagnostic criteria for PTSD or anxiety or depression [11][12][14][17].

\section{Limitations of the Study}

This case series has a small sample size and therefore the findings cannot be generalized to a wider population.

\section{Findings in Relation to other Research}

Whilst this study is based on a small sample size, the results perhaps highlight how effective EMDR can be in routine community settings. The findings support case studies in the United States[18][19]which also suggest EMDR is an effective treatment for rape trauma. In addition, findings support wider research demonstrating EMDR as an effective treatment for PTSD [5]. What is particularly interesting to note here is how, in one case, EMDR was used successfully to treat multiple childhood sexual abuse traumas with just one session per traumatic memory. This may offer a useful and efficient treatment of childhood sexual abuse trauma.

The study results suggest that large scale, randomized, control trials and cohort studies are now required, to provide an enhanced evidence base so that more clients experiencing sexual trauma are able to access effective treatment, through EMDR.

\section{Conclusion}

Whilst this is a small sample, case series design, the research findings highlight that EMDR is an efficient and effective treatment of both adult and childhood sexual abuse trauma. Large scale, randomised, control studies are now required to further investigate the effectiveness and speed of treatment of EMDR, so that more clients are able to access EMDR therapy following experience of sexual trauma.

\section{Reference}

1. Dube SR, Anda RF, Felitti VJ, Chapman DP, Williamson DF, et al. (2001) Childhood abuse, household dysfunction, and the risk of attempted suicide throughout the life span: findings from the Adverse Childhood Experiences Study. Jama 286(24): 3089-3096.

2. Elliott DM, Mok DS, Briere J (2004) Adult sexual assault: Prevalence, symptomatology, and sex differences in the general population. Journal of traumatic stress 17(3): 203-211.
3. Patterson D, Greeson M, Campbell R (2009) Understanding rape survivors' decisions not to seek help from formal social systems. Health \& Social Work 34(2): 127-136.

4. National Institute for Clinical Excellence NICE (2005) The management of Post-Traumatic Stress Disorder stress disorder in primary and secondary care. London.

5. Bisson JI, Ehlers A, Matthews R, Pilling S, Richards D, et al. (2007) Psychological treatments for chronic post-traumatic stress disorder. The British journal of psychiatry 190(2): 97-104.

6. Shapiro, F. (1989). Eye movement desensitization: A new treatment for post-traumatic stress disorder. Journal of behavior therapy and experimental psychiatry, 20(3), 211-217.

7. Ehlers A, Clark DM (2000) A cognitive model of posttraumatic stress disorder. Behaviour research and therapy 38(4): 319-345.

8. Litz BT, Gray MJ, Bryant RA, Adler AB (2002) Early intervention for trauma: Current status and future directions. Clinical psychology: science and practice 9(2): 112-134.

9. Kessler BL, Bieschke KJ (1999) A retrospective analysis of shame, dissociation, and adult victimization in survivors of childhood sexual abuse. Journal of Counseling Psychology 46(3): 335.

10. Blore DC, Holmshaw EM, Swift A, Standart S, Fish DM (2013) The development and uses of the "blind to therapist" EMDR protocol. Journal of EMDR Practice and Research 7(2): 95-105.

11. Beck AT, Steer RA, Brown GK (1996) Beck depression inventory-II. San Antonio 78(2): 490-8.

12. Beck AT, Steer RA (1990) Manual for the Beck anxiety inventory. Psychological Corporation, San Antonio, Texas, USA.

13. Lambert MJ, Burlingame GM, Umphress V, Hansen NB, Vermeersch DA, et al. (1996) The reliability and validity of the Outcome Questionnaire. Clinical Psychology and Psychotherapy 3(4): 249-258.

14. Creamer M, Bell R, Falila S (2002) Psychometric properties of the Impact of Events Scale-Revised. Behaviour Research and Therapy 41: 1489-1496.

15. Shapiro F (2014) The role of eye movement desensitization and reprocessing (EMDR) therapy in medicine: addressing the psychological and physical symptoms stemming from adverse life experiences. The Permanente Journal 18(1): 71.

16. Shapiro F (2001) Eye movement desensitization and reprocessing: Basic principles, protocols and procedures. (2nd Edn.), Guilford, New York, USA.

17. American Psychiatric Association (2013) Diagnostic and statistical manual of mental disorders (5th Edn.), DC: American Psychiatric, Washington, US.

18. Shapiro F (2002) EMDR 12 years after its introduction: past and future research. Journal of clinical psychology 58(1): 1-22.

19. Edmond T, Rubin A, Wambach KG (1999) The effectiveness of EMDR with adult female survivors of childhood sexual abuse. Social Work Research 23(2): 103-116. 
This work is licensed under Creative Commons Attribution 4.0 License

DOI: 10.19080/PBSIJ.2017.06.555676
Your next submission with Juniper Publishers will reach you the below assets

- Quality Editorial service

- Swift Peer Review

- Reprints availability

- E-prints Service

- Manuscript Podcast for convenient understanding

- Global attainment for your research

- Manuscript accessibility in different formats ( Pdf, E-pub, Full Text, Audio)

- Unceasing customer service

Track the below URL for one-step submission https://juniperpublishers.com/online-submission.php 